\title{
Inpatient bedspacing: could a common response to hospital crowding cause increased patient mortality?
}

\author{
Finlay A McAlister, ${ }^{1}$ Kaveh G Shojania ${ }^{2}$
}

- Additional material is published online only. To view please visit the journal online (http://dx.doi.org/10.1136/ bmjqs-2017-007524).

'Division of General Internal Medicine, University of Alberta, Edmonton, Alberta, Canada ${ }^{2}$ Department of Medicine, University of Toronto, Toronto, Ontario, Canada

\section{Correspondence to}

Dr Finlay A McAlister, Division of General Internal Medicine, University of Alberta, Edmonton, Alberta T6G2R7, Canada; finlay.mcalister@ualberta.ca

Accepted 27 October 2017 Published Online First 27 November 2017

\section{SLinked}

- http://dx.doi.org/10.1136/ bmjqs-2017-006925

CrossMark

To cite: McAlister FA, Shojania KG. BMJ Qual Saf 2018;27:1-3.
Emergency department (ED) overcrowding results in patient and provider dissatisfaction, poorer quality of care, increased healthcare costs, and even increased mortality in some studies. ${ }^{1-4}$ In response to this evidence, many hospitals have instituted full capacity protocols in which patients in the ED who are admitted but waiting for a bed on the home ward of the admitting service are sent to the first available inpatient bed (or even inpatient hallway) even if it is off-service-a practice known in the UK as boarding or medical outliers, ${ }^{56}$ and in other countries as bedspacing.

Why might bedspacing matter? Caring for these patients may seem to present only the minor inconvenience to physicians of making short trips off their home ward to visit the floor of some other clinical service. The potential problem arises with undermining multidisciplinary care. While physician care is delivered by the admitting service, the off-service ward staff provide nursing care, pharmacy medication reconciliation, physiotherapy, swallowing assessments, occupational therapy and social work support. These different services tend to function more cohesively when the individual health professionals involved can interact face to face and, even more importantly, know each other well. Bedspacing thus separates the physicians caring for the patient from the other health professionals on the patient's care team. Thus, one might well expect difficulties with care coordination for bedspaced patients, manifesting as longer hospital lengths of stay in at least one study. ${ }^{6}$

Despite the enthusiasm of system planners and ED policy makers for bedspacing, evidence for this approach remains surprisingly scant. ${ }^{7}$ Indeed, a systematic review identified only one published study of weak methodological quality (a single-centre before-after study) and the only outcomes evaluated were ED-specific time metrics. The authors of the systematic review uncovered several positive testimonials about bedspacing in the grey literature, but cautioned that 'there has not been a strong documentation of potential harms or drawbacks of such full capacity protocol strategies'? Thus, the study by Bai and colleagues in this issue of BMJ Quality and Safety evaluating outcomes in bedspaced general internal medicine (GIM) patients makes a particularly useful contribution to the literature. $^{8}$

After excluding patients who were assigned to short-stay or step-down units, transferred to other hospital services, left against medical advice, or died while still in the ED, Bai and colleagues found that one-third of GIM admissions were bedspaced to off-service wards (varying from $19 \%$ to $47 \%$ daily). Surprisingly, these patients had higher inpatient mortality ( $8 \%$ compared with $4 \%$ ) than patients admitted to GIM home wards. It is less surprising that the excess mortality risk associated with bedspacing was highest in the first week of hospitalisation since that is when patients are most medically active. The strength of association was similar across strata defined by Charlson comorbidity scores and whether bedspacing was onto surgical or medical off-service wards. The association remained robustly significant after adjustment for demographics, clinical variables, and time-dependent covariates 
such as capacity ratios (a proxy for hospital occupancy and team workload), and also across a number of sensitivity analyses using Cox proportional hazards, competing risk models, propensity score matching, and matching on factors known to impact outcomes such as attending team, day of admission and case mix group. ${ }^{9-11}$

Not having data on length of time in the ED prior to ward transfer represents a weakness of this study. But the fact that over $85 \%$ of the deaths occurred 2 days or more after admission somewhat mitigates the concern that differences in time spent and care provided in the ED drove the poorer outcomes for bedspaced patients. It is important to also note that only $11 \%$ of patients were moved during their hospitalisation $(6 \%$ from off-service to a GIM home ward; 5\% from a GIM ward to off-service), and results were similar whether bedspacing was defined based on initial ward assignment or ward at the time of discharge.

While the retrospective cohort design of the study by Bai and colleagues means that we cannot definitely attribute causality, the robustness of their findings across multiple sensitivity analyses and the relatively large sample size support the internal validity of their findings: bedspaced GIM patients in their institution in the years studied exhibited higher mortality rates than GIM patients admitted to GIM wards. We can never confidently rule out the effects of unmeasured confounders in any observational study. In this study, for instance, severity of illness could not be compared between bedspaced and home ward patients. That said, it is not unreasonable to assume that any systematic bias in the assignment of GIM patients from the ED would have favoured the placement of sicker patients on to home wards rather than off-service-a practice that should have influenced mortality outcomes in the opposite direction to the exposure effect documented by Bai and colleagues.

Is the magnitude of the effect size plausible? Could bedspacing actually confer a greater risk to patients than being admitted on a weekend or at the beginning of the academic year? ${ }^{10}{ }^{11}$ While the authors provide several potential explanations for the apparent mortality risk arising from bedspacing, the reality is that we are never short of theories to explain observational data. In fact, an online supplementary appendix provides excerpts from the peer review process, including comments from the Editor (KGS), one of the reviewers (FAM) and the authors. These excerpted comments highlight both the degree to which one can offer competing explanations for observational results and the hypothesis-generating nature of the striking and alarming results reported by Bai and colleagues. ${ }^{8}$

As the authors themselves point out, the medical literature contains numerous examples of single-centre observational studies suggesting large exposure effects but which were subsequently overturned by stronger, multisite studies. While the Charlson scores, length of stay and in-hospital mortality rates in their study are similar to those reported from other Canadian university hospitals with similar GIM service distributions, ${ }^{12}$ this study definitely needs to be replicated in other settings. While one American study ${ }^{13}$ reported that localising GIM patients to home wards improved workflow efficiency but increased length of stay and mortality, we $\mathrm{e}^{12}$ found that cohorting our GIM patients on home wards rather than having them bedspaced throughout our institution resulted in substantially shorter lengths of stay without any impact on mortality - similar to a study in a UK district general hospital. ${ }^{6}$ Interestingly, we also found no impact on ED wait times or crowding metrics, suggesting that the potential benefits of inpatient bedspacing for EDs are smaller than assumed. ${ }^{14}$

On the one hand, we must view the results of Bai and colleagues as hypothesis-generating and requiring confirmation in other settings, as must happen with any important finding arising from a single-centre observational study. On the other hand, the alarming findings from their robust analysis should concern all of us and, at the very least, prompt a re-examination of the assumption that bedspacing represents a riskfree policy option. In 2014, the College of Emergency Medicine in the UK endorsed bedspacing as one of the solutions to ED crowding: 'the guideline development group acknowledged that whilst boarding was based on weak evidence it was the option associated with least risk'. ${ }^{15}$ The study by Bai et al should stimulate further research into the risk-benefit ratio of bedspacing and, more generally, remind us all of the need for evidence to inform policy decisions.

Contributors FAM and KGS contributed to the conception of the paper; they critically read and modified subsequent drafts and approved the final version. KGS is an editor at BMJ Quality and Safety.

Competing interests None declared.

Provenance and peer review Commissioned; internally peer reviewed.

(C) Article author(s) (or their employer(s) unless otherwise stated in the text of the article) 2018. All rights reserved. No commercial use is permitted unless otherwise expressly granted.

\section{REFERENCES}

1 Bernstein SL, Aronsky D, Duseja R, et al. The effect of emergency department crowding on clinically oriented outcomes. Acad Emerg Med 2009;16:1-10.

2 Guttmann A, Schull MJ, Vermeulen MJ, et al. Association between waiting times and short term mortality and hospital admission after departure from emergency department: population based cohort study from Ontario, Canada. BMJ 2011;342:d2983.

3 Richardson DB. Increase in patient mortality at 10 days associated with emergency department overcrowding. Med J Aust 2006;184:213-6.

4 Sun BC, Hsia RY, Weiss RE, et al. Effect of emergency department crowding on outcomes of admitted patients. Ann Emerg Med 2013;61:605-11. 
5 Boyle A, Viccellio P, Whale C. Is "boarding" appropriate to help reduce crowding in emergency departments? BMJ 2015;350:h2249.

6 Stylianou N, Fackrell R, Vasilakis C. Are medical outliers associated with worse patient outcomes? A retrospective study within a regional NHS hospital using routine data. BMJ Open 2017;7:e015676

7 Villa-Roel C, Guo X, Holroyd BR, et al. The role of full capacity protocols on mitigating overcrowding in EDs. Am J Emerg Med 2012;30:412-20.

8 Bai AD, Srivastava S, Tomlinson GA, et al. Mortality of hospitalised internal medicine patients bedspaced to noninternal medicine inpatient units: retrospective cohort study. BMJ Qual Saf 2018;27:11-20.

9 Eriksson CO, Stoner RC, Eden KB, et al. The association between hospital capacity strain and inpatient outcomes in highly developed countries: a systematic review. J Gen Intern Med 2017;32:686-96.

10 Bell CM, Redelmeier DA. Mortality among patients admitted to hospitals on weekends as compared with weekdays. N Engl J Med 2001;345:663-8.
11 Young JQ, Ranji SR, Wachter RM, et al. "July effect": impact of the academic year-end changeover on patient outcomes: a systematic review. Ann Intern Med 2011;155:309-15.

12 McAlister FA, Bakal JA, Majumdar SR, et al. Safely and effectively reducing inpatient length of stay: a controlled study of the General Internal Medicine Care Transformation Initiative. BMJ Qual Saf 2014;23:446-56.

13 Singh S, Tarima S, Rana V, et al. Impact of localizing general medical teams to a single nursing unit. J Hosp Med 2012;7:551-6.

14 McAlister FA, Bakal JA, Rosychuk RJ, et al. Does reducing inpatient length of stay have upstream effects on the emergency room: exploring the impact of the general internal medicine care transformation initiative. Acad Emerg Med 2016;23:711-7.

15 Boyle A, Higginson I, Smith S, et al. Crowding in emergency departments. London, United Kingdom: Royal College of Emergency Medicine, 2014. https://www.rcem.ac.uk/docs/ Service\%20Design\%20+\%20Delivery/52b.\%20RCEM\% 20Crowding\%20Guideline $\% 20$ 